\title{
A benzene vapor sensor with avoiding humidity false response based on metal-organic framework-modified quartz crystal microbalance
}

\author{
Zhiheng Ma, Zhixuan Cheng, Qun Xiang, Xiaohong Wang, Jiaqiang $X u^{*}$ \\ NEST Lab, Department of chemistry, College of Science, Shanghai University, Shanghai, 200444, \\ China. \\ *E-mail: xujiaqiang@shu.edu.cn
}

\begin{abstract}
:
As one of typical VOCs, benzene is seriously harmful to human health. There is really lack of portable sensing method to directly detect benzene in air duo to its chemical inertness. Herein the metalorganic framework(MOF) of MOF-14(Cu $(\mathrm{CTB})_{2}\left(\mathrm{H}_{2} \mathrm{O}\right)_{3} 2(\mathrm{DMF}) 9\left(\mathrm{H}_{2} \mathrm{O}\right)_{2}$ MOF-14 is first explored for sensing to the nonpolar molecule of benzene. In previous studies, adsorption experiments all use the dry gas that neglect the influence of humidity, so we used a new method to detect benzene in order to avoid water vapor false response for practical use. By loading MOFs on quartz crystal microbalance(QCM), sensing experiments for four MOFs of MOF-177, HKUST-1, MOF-74, and MOF14 approve that MOF-14 has a high selectivity, repeatability, long-term stability for benzene detection. The adsorption mechanism of benzene on sensor was identified that is via host-guest interaction of MOF with benzene molecule and studied by Van der Waals corrected density functional theory calculation.
\end{abstract}

Key words: MOF-14, QCM, Benzene, Sensor

\section{Results and Discussion of benzene sensing performance}

As is shown in Fig.1, it is obviously seen that MOF-14 based QCM sensor shows a good selectivity to benzene vapor. when QCM sensor exposes to tested vapors(all at $100 \mathrm{ppm}$ ), it shows the highest frequency change of $1500 \mathrm{~Hz}$ to benzene, and the lowest response $(200 \mathrm{~Hz})$ to methanol. The preferential selectivity towards benzene may in dicate the $\pi-\pi$ interaction and Lewis acidity. However, to the other three interfering vapors of toluene, ethylbenzene and xylene ,the molecules of toluene or xylene also provide similar host-guest interaction with the MOF-14 sensing material. Due to the greater steric hindrance of xylene or toluene than benzene,the sensor outputs lower response to toluene or xylene than benzene of identical concentration.

Fig.2 summarizes the detection results of the four MOF-loaded sensors to benzene vapor with identical concentration of 60ppm. It can be clearly seen that MOF-14 exhibits the highest sensing response among the four MOFs. All of four MOFs have similar carboxyl-based linker. MOF-14 and HKUST-1 are constructed with $\mathrm{Cu}^{2+}$ units, thus, has more moderate Lweis acidity than MOF-177 and MOF-74 (respectively with $\mathrm{Zn}^{2+}$ and $\mathrm{Mg}^{2+}$ as building blocks). Compared with HKUST-1, the more benzene ring structural of MOF-14 should be a better material to detect xylene, due to the $\pi-\pi$ interaction.

Fig. 3 shows the room-temperature real-time sensing curve of the MOF-14 to $20 \mathrm{ppm}$ benzene vapors with various relative humidity $(\mathrm{RH})$ of $22 \%-100 \%$. As is shown in the inset of Fig.3, the sensor outputs $40 \mathrm{~Hz}$ response to the pure water vapor of $45 \mathrm{RH} \%$. Compared with 20 ppm benzene vapor at 45 $\mathrm{RH} \%$, the response is $250 \mathrm{~Hz}$. The sensing signal of $20 \mathrm{ppm}$ benzene vapor at different relative huimidity is much higher than pure water vapor. Thus we conclude that water may play a important role in the sensing to benzene vapor.

Fig.4 shows the response curves of QCM gas sensors to different benzene concentrations at $93 \mathrm{RH} \%$. The curves shows that at high relative humidity the response of benzene vapor is very large. Especially the response of $20 \mathrm{ppm}$ benzene is several times to that of water at 93 $\mathrm{RH} \%$. 
Fig.5 shows the response curves of low concentration benzene at low relative humidity. The sensors outputs $80 \mathrm{~Hz}$ response to the benzene vapor of $80 \mathrm{ppm}$. The $80 \mathrm{~Hz}$ sensing signal is much higher than the noise-floor of about $5 \mathrm{~Hz}$. Thus we conclude that the MOF-14 based benzene sensor features the very fine limit of detection (LOD) of $274 \mathrm{ppb}$, which is already lower than the human olfactory threshold of $470 \mathrm{ppb}$.

In summary, benzene sensing performances of MOF materials have been systematically investigated by using the QCM. According to research, the typical MOF material of MOF-14 shows satisfactory sensing performance to trace benzene vapor. Especially the water resistance make it possible to be a practical sensor.

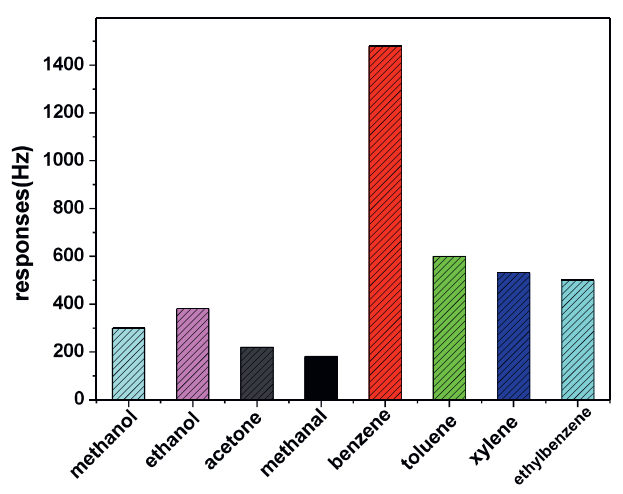

Fig. 1 Response of the MOF-14 sensor to 12 kinds of vapors for selectivity

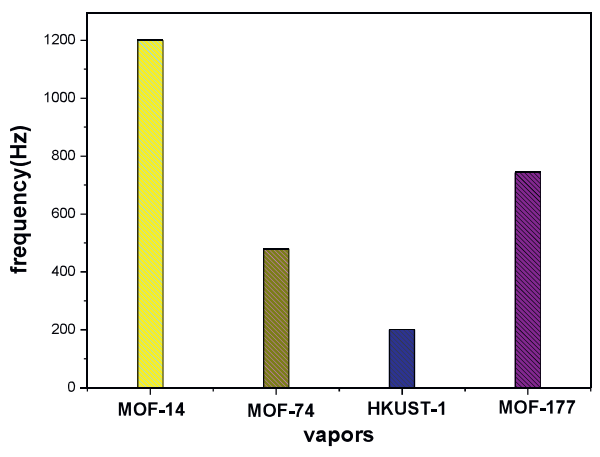

Fig.2 QCM-sensing results of four kinds of MOFs to benzene vapor with an identical concentration of 60 ppm

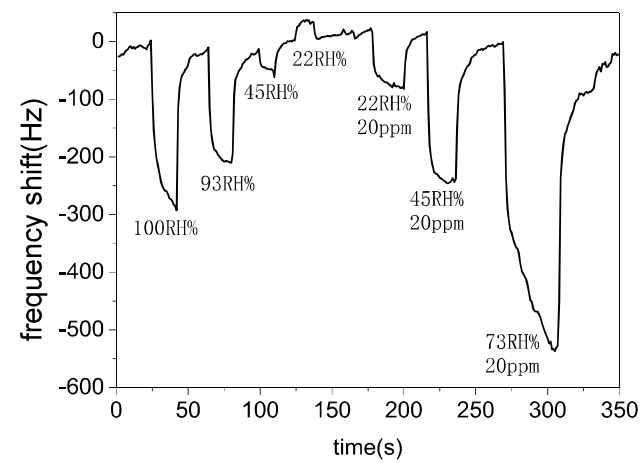

Fig.3 Response curves of QCM gas sensor to the different relative humidity

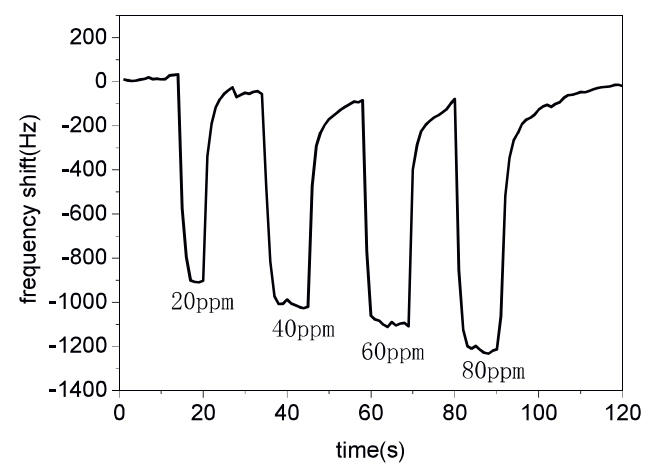

Fig.4. Response curves of QCM gas sensor to the different concentrations of benzene at $93 \mathrm{RH} \%$

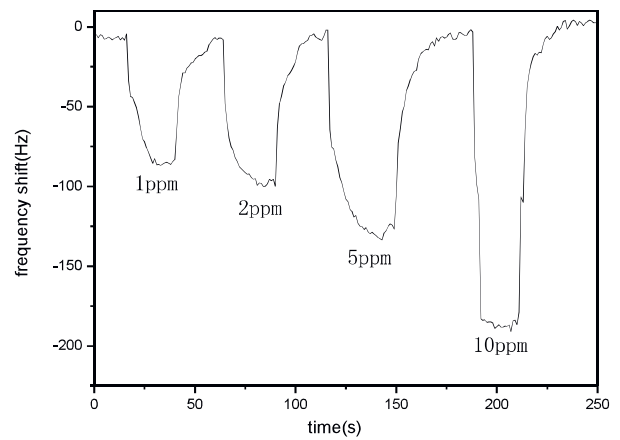

Fig. 5 Sensing responses versus various concentrations of benzene vapor at $22 \mathrm{RH} \%$

\section{Reference}

[1] Bearzotti, Andrea, et al. "A study of a QCM sensor based on pentacene for the detection of BTX vapors in air." Sensors \& Actuators B Chemical 240(2017):1160-1164. DOI: 10.1016/j.snb.2016.09.097

[2] Xu, Fen, et al. "A pyridine vapor sensor based on metal-organic framework-modified quartz crystal microbalance." Sensors \& Actuators B Chemical 254(2017):872-877. DOI: 10.1016/j.snb.2017.07.026 